\title{
Mutational Analysis of an Antigenic Peptide Shows Recognition in a Loop Conformation
}

\author{
Philippe Rondard, Michel E. Goldberg, and Hugues Bedouelle* \\ Unité de Biochimie Cellulaire, CNRS URA 1129, Institut Pasteur, 28 rue du Docteur Roux, 75724 Paris Cedex 15, France
}

Received October 7, 1996; Revised Manuscript Received May 8, $1997^{\otimes}$

\begin{abstract}
We have analyzed the recognition between an antigenic undecapeptide and a monoclonal antibody through a mutational approach. Antibody mAb164 is directed against the native form of the $\mathrm{TrpB}_{2}$ subunit of Escherichia coli tryptophan synthase. It recognizes a synthetic peptide, P11, constituted of residues 273-HGRVGIYFGMK-283 of TrpB with high affinity. P11 was fused with a carrier protein, MalE, to facilitate its manipulation. The affinities between mAb164 and the MalE-P11 hybrids were measured by competition enzyme-linked immunosorbent assay (ELISA). The changes of the P11 residues into progressively shorter residues, the comparison of changes into Pro and Ala, and the study of double mutants showed the following. Four hydrophobic residues of P11, Val276, Ile278, Tyr279, and Phe280, were predominant in the interaction. For some residues, e.g., Tyr279, most groups of the side chain contributed to the interaction. For others, only some groups played a significant role, e.g., the $\mathrm{C}_{\delta}$ group of Ile278 or the $\mathrm{C}_{\beta}$ group of Phe280. The lack of side chain in position Gly281 and a tertiary interaction between the side chains of Ile 278 and Lys 283 were important. P11 was recognized in a loop conformation, close to that of residues $273-283$ of TrpB in the crystal structure of the complete tryptophan synthase, $\operatorname{TrpA}_{2} \operatorname{TrpB}_{2}$. Comparison of our mutational data with NMR data on the conformation of the isolated peptide P11 and with kinetic data on its interaction with mAb164 indicate that mAb164 selects a conformer of P11 that represents only a small minority of the molecules. Our results provide useful information on the mechanisms by which linear epitopes and unconstrained peptides are recognized by receptors.
\end{abstract}

Many essential biological processes involve interactions between peptides and proteins (Marshall, 1992; Zvelebil, 1993; Stanfield \& Wilson, 1995) and short peptides are often used to mimic one protein in studies of protein-protein interactions (Scott \& Smith, 1990; Cortese et al., 1995). Therefore, a better understanding of the peptide-protein interactions should improve our ability to design or select peptides for use as synthetic vaccines or new pharmaceuticals (Clackson \& Wells, 1994; Houghten, 1993; Liuzzi et al., 1994; Divita et al., 1995).

We studied the recognition of an undecapeptide by an antibody through a mutational approach. mAb164 is a mouse monoclonal antibody that is directed against one subunit (the homodimer $\left.\operatorname{TrpB}_{2}\right)^{1}$ of the tryptophan synthase from $E s$ cherichia coli. The equilibrium dissociation constant, $K_{\mathrm{D}}$, of their complex is equal to $1 \mathrm{nM}$ (Friguet et al., 1989a). mAb164 recognizes a synthetic peptide, which is constituted of residues 273-283 of $\operatorname{TrpB}$ and called $\mathrm{P} 11$, with high affinity $\left(K_{\mathrm{D}}=7 \mathrm{nM}\right)$ (Larvor et al., 1991). The crystal structure of the complete tryptophan synthase (the heterotetramer $\operatorname{Trp} A_{2} \operatorname{Trp} B_{2}$ ) is known but not the structure of the free form of $\operatorname{TrpB}_{2}$, which was used as immunogen to raise mAb164. Residues 273-283 of TrpB form a hairpin in the structure of $\operatorname{Trp}_{2} \mathrm{TrpB}_{2}$ but eight of them belong to the interface between $\operatorname{TrpA}$ and $\operatorname{TrpB}_{2}$ so that their conforma-

* Corresponding author: Tel 33-1-45 6883 79; fax 33-1-40 6130 43; email hbedouel@pasteur.fr.

${ }^{\otimes}$ Abstract published in Advance ACS Abstracts, July 1, 1997.

${ }^{1}$ Abbreviations: MalE, maltose binding protein, product of the malE gene of Escherichia coli; $\operatorname{TrpA}$ and $\operatorname{TrpB}$, subunits of tryptophan synthase, products of the $\operatorname{trp} A$ and $\operatorname{trp} B$ genes of $E$. coli; $\operatorname{TrpA}_{2} \operatorname{TrpB}_{2}$, tryptophan synthase; ELISA, enzyme-linked immunosorbent assay; NMR, nuclear magnetic resonance. tions could be different in $\operatorname{Trp} \mathrm{A}_{2} \operatorname{Trp} \mathrm{B}_{2}$ and in the free $\operatorname{Trp} \mathrm{B}_{2}$ (Hydes et al., 1988; Rhee et al., 1996). A conformational analysis of the isolated synthetic peptide P11 by proton NMR spectroscopy has shown that its molecules, in majority, adopt an extended conformation but that some of them, in minority, are structured in their C-terminal part and comprise at least two different conformers (Delepierre et al., 1991). For all these reasons, the conformation of P11 that is recognized by mAb164 and the mechanism of this recognition remained unknown.

To solve this problem, we constructed mutant derivatives of P11 and compared their affinities for antibody mAb164 with that of the wild-type peptide. Rather than chemically synthesizing the mutant derivatives of P11, we chose to introduce the corresponding mutations at the genetic level into a fusion protein, MalE-P11, in which the C-terminal end of protein MalE was linked with the N-terminal end of P11. We have previously shown that the wild-type hybrid, MalE-P11(wt), can be produced in E. coli from a plasmidic gene and purified in one step as MalE. A comparison of the proton NMR spectra of MalE-P11(wt), MalE, and P11 has shown that the fusion between these two last polypeptides does not constrain the conformation of P11. The purified hybrid MalE-P11(wt) and the synthetic peptide P11 have identical affinities for mAb164 (Rondard et al., 1997). Thus, MalE-P11 could be used to study the recognition between mAb164 and P11 by a genetic and mutational approach.

\section{MATERIALS AND METHODS}

Production and Purification of the MalE-P11 Hybrids. The construction of plasmid pPR1, which codes for hybrid 
MalE-P11, the mutagenesis of pPR1 with oligonucleotides, the production of the MalE-P11 hybrids in E. coli, their purification by affinity chromatography on cross-linked amylose, the analysis of the purified preparations by electrophoresis through SDS-polyacrylamide gels and the measurement of the protein concentrations were performed as described (Rondard et al. 1997 (preceding paper in this issue)). The purified preparations of the mutant hybrids were not degraded and were homogeneous at $>95 \%$, as previously found for the wild type.

Equilibrium Dissociation Constants. The equilibrium dissociation constants, $K_{\mathrm{D}}$, between antibody mAb164 and the MalE-P11 hybrids were measured by a competition ELISA (Friguet et al., 1989a,b) with modifications in the mathematical treatment of the raw measurements. The measurements were performed at $25^{\circ} \mathrm{C}$ in $50 \mathrm{mM}$ Tris- $\mathrm{HCl}$, pH 7.5, $50 \mathrm{mM} \mathrm{NaCl}, 10 \mathrm{mM}$ maltose, and $0.02 \%$ bovine serum albumin. mAb164 and the antigen were first incubated together in solution during $15 \mathrm{~h}$, i.e. until equilibrium was reached. The concentration of antigen was high enough to fully saturate mAb164 and thus avoid errors due to the bivalency of the antibody (Stevens, 1987). The concentration of free mAb164 was then determined by a direct ELISA in which the apo form of protein $\operatorname{TrpB}_{2}$ coated the wells of the microtitration plate. The coating of the wells was performed with a $1 \mu \mathrm{g} / \mathrm{mL}$ solution of $\operatorname{TrpB}_{2}$. The bound mAb164 was revealed with a goat antibody directed against mouse IgG$(\mathrm{H}+\mathrm{L})$, conjugated with alkaline phosphatase and affinitypurified (Promega). Each ELISA measurement was made in triplicate and in conditions such that $i=i_{\mathrm{t}}\left(A / A_{0}\right)$, where $i$ is the concentration of free antibody, $i_{\mathrm{t}}$ is the total concentration of antibody, $A$ is the absorbance of the sample at $405 \mathrm{~nm}$, and $A_{0}$ is the absorbance for a sample with no antigen present. If $a$ is the concentration of free antigen, $a_{\mathrm{t}}$ is the total concentration of antigen, and $x$ is the concentration of complex between antibody and antigen, then $x=i_{\mathrm{t}}-i$ and $a=a_{\mathrm{t}}-x=a_{\mathrm{t}}-\left(i_{\mathrm{t}}-i\right)$. By replacing $a$ and $x$ by their expressions as functions of $i$ in the law of mass action $K_{\mathrm{D}}=a i / x$, where $K_{\mathrm{D}}$ is the dissociation constant between antibody and antigen, one obtains the following equation: $A=A_{0}\left\{i_{\mathrm{t}}-a_{\mathrm{t}}-K_{\mathrm{D}}+\left[\left(i_{\mathrm{t}}-a_{\mathrm{t}}-K_{\mathrm{D}}\right)^{2}+4 K_{\mathrm{D}} i_{\mathrm{t}}\right]^{1 / 2}\right\} / 2 i_{\mathrm{t}}$ when $i_{\mathrm{t}}<a_{\mathrm{t}}+K_{\mathrm{D}}$. This equation was directly fitted to the raw experimental values of $A$, taking $K_{\mathrm{D}}$ as a fitting parameter. The $K_{\mathrm{D}}$ measurements were repeated at least three times, in independent experiments.

Structural Analysis. We used the refined atomic coordinates of $\operatorname{TrpA}_{2} \operatorname{TrpB}_{2}$ with bound $\mathrm{Na}^{+}$(Hydes et al., 1988; C. C. Hydes, K. D. Parris, T. N. Bhat, C. Brown, S. A. Ahmed, E. W. Miles, and D. R. Davies, manuscript in preparation). The side chains of residues Tyr279 and Phe280 of TrpB have different conformations when $\mathrm{Na}^{+}$is replaced by $\mathrm{K}^{+}$or $\mathrm{Cs}^{+}$(Rhee et al., 1996). The solvent-accessible surface areas were calculated as described, using a sphere probe of $1.4 \AA$ in diameter (Shrake \& Ruplay, 1973; Koehl \& Delarue, 1994).

\section{RESULTS AND DISCUSSION}

We measured the equilibrium dissociation constant, $K_{\mathrm{D}}$, at $25^{\circ} \mathrm{C}$ between mAb164 and either MalE-P11(wt) or each of its mutant derivatives by a method of competition ELISA and calculated the corresponding free energy of dissociation, $\Delta G$ (Table 1). We then compared the differences, $\Delta \Delta G$,
Table 1: Equilibrium Constants and Associated Free Energies for the Dissociation between mAb164 and the MalE-P11 Derivatives at $25{ }^{\circ} \mathrm{C}^{a}$

\begin{tabular}{|c|c|c|c|}
\hline mutation & $\begin{array}{c}K_{\mathrm{D}} \pm \mathrm{SE} \\
(\mathrm{nM})\end{array}$ & $\begin{array}{c}\Delta G \pm \mathrm{SE} \\
(\mathrm{kcal} / \mathrm{mol})\end{array}$ & $\begin{array}{c}\Delta \Delta G \pm \mathrm{SE} \\
(\mathrm{kcal} / \mathrm{mol})\end{array}$ \\
\hline wt & $5.2 \pm 0.8$ & $11.35 \pm 0.10$ & $0.0 \pm 0.1$ \\
\hline H273A & $3.7 \pm 0.6$ & $11.50 \pm 0.10$ & $-0.2 \pm 0.1$ \\
\hline G274A & $44 \pm 8$ & $10.03 \pm 0.10$ & $1.3 \pm 0.1$ \\
\hline R275A & $4.9 \pm 0.5$ & $11.34 \pm 0.06$ & $0.0 \pm 0.1$ \\
\hline V276A & $3100 \pm 800$ & $7.53 \pm 0.15$ & $3.8 \pm 0.2$ \\
\hline V276P & $15000 \pm 8000$ & $6.83 \pm 0.31$ & $4.5 \pm 0.3$ \\
\hline G277A & $16 \pm 13$ & $11.09 \pm 0.53$ & $0.3 \pm 0.5$ \\
\hline G277P & 370000 & 4.68 & 6.7 \\
\hline I278A & $47000 \pm 27000$ & $6.27 \pm 0.56$ & $5.1 \pm 0.6$ \\
\hline I278V & $51000 \pm 4000$ & $5.85 \pm 0.02$ & $5.5 \pm 0.1$ \\
\hline I278P & $940 \pm 190$ & $8.26 \pm 0.13$ & $3.1 \pm 0.2$ \\
\hline Y279A & $12000 \pm 5000$ & $6.75 \pm 0.25$ & $4.6 \pm 0.3$ \\
\hline Y279F & $85 \pm 65$ & $10.16 \pm 0.62$ & $1.2 \pm 0.6$ \\
\hline Y279L & $260 \pm 30$ & $9.00 \pm 0.08$ & $2.4 \pm 0.1$ \\
\hline Y279P & $150000 \pm 50000$ & $5.27 \pm 0.23$ & $6.1 \pm 0.3$ \\
\hline F280A & $56 \pm 14$ & $9.93 \pm 0.14$ & $1.4 \pm 0.2$ \\
\hline F280G & $1800 \pm 100$ & $7.84 \pm 0.04$ & $3.5 \pm 0.1$ \\
\hline F280L & $2100 \pm 200$ & $7.73 \pm 0.04$ & $3.6 \pm 0.1$ \\
\hline F280P & $5300 \pm 800$ & $7.21 \pm 0.08$ & $4.1 \pm 0.1$ \\
\hline G281A & $350 \pm 90$ & $8.86 \pm 0.15$ & $2.5 \pm 0.2$ \\
\hline G281P & $4400 \pm 600$ & $7.31 \pm 0.08$ & $4.0 \pm 0.1$ \\
\hline M282A & $130 \pm$ & 9.45 & $1.9 \pm 0.1$ \\
\hline M282G & $310 \pm 20$ & $8.87 \pm 0.05$ & $2.5 \pm 0.1$ \\
\hline M282P & $5900 \pm 2200$ & $7.21 \pm 0.20$ & $4.1 \pm 0.2$ \\
\hline K283A & $190 \pm 80$ & $9.21 \pm 0.26$ & $2.1 \pm 0.3$ \\
\hline $\mathrm{K} 283 \mathrm{G}$ & $160 \pm 40$ & $9.28 \pm 0.14$ & $2.1 \pm 0.2$ \\
\hline K283M & $27 \pm 9$ & $10.39 \pm 0.21$ & $1.0 \pm 0.2$ \\
\hline V276A,K283A & $170000 \pm 90000$ & $5.49 \pm 0.38$ & $5.9 \pm 0.4$ \\
\hline $\mathrm{I} 278 \mathrm{~V}, \mathrm{~K} 283 \mathrm{~A}$ & $2600 \pm 700$ & $7.67 \pm 0.17$ & $3.7 \pm 0.2$ \\
\hline
\end{tabular}

${ }^{a}$ The residues of the P11 moiety of MalE-P11 are numbered according to their positions in the sequence of TrpB. I278V, change in the P11 moiety of MalE-P11 replacing the wild-type side chain, Ile, at position 278 by Val; I278V,K283A, a double change; wt, no change; mut, one of the changes of the first column. The mean values and associated standard errors (SE) of $K_{\mathrm{D}}, \Delta G=-R T \ln K_{\mathrm{D}}$, and $\Delta \Delta G$ $=\Delta G(\mathrm{wt})-\Delta G(\mathrm{mut})$ in three independent experiments are given. The standard error on $\Delta \Delta G$ was calculated from the standard errors on $\Delta G(\mathrm{wt})$ and $\Delta G$ (mut) through the formula $[\operatorname{SE}(\Delta \Delta G)]^{2}=$ $[\operatorname{SE}(\Delta G(\mathrm{wt}))]^{2}+[\mathrm{SE}(\Delta G(\text { mut }))]^{2}$. MalE-P11 contained 382 residues. Residues 1-369 came from MalE, residues 370 and 371 (Arg-Ile) formed a linker, and residues 372-382 came from P11. Changing the arginine of the linker into Ala did not affect the properties of the R275A mutation.

between the $\Delta G$ values to analyze the contributions of the different chemical groups of P11 to its interaction with $\mathrm{mAb} 164$, either by a direct contact or through an intramolecular conformational effect. In the following, the residues of P11 are numbered according to their position in the sequence of TrpB: His273-Gly274-Arg275-Val276-Gly277Ile278-Tyr279-Phe280-Gly281-Met282-Lys283.

Deletion of the Side Chains into Ala or Gly. The residues of P11 were first mutated into Ala or Gly to delete their side chains. These mutations showed that the side chains of four residues, Val276, Ile278, Tyr279, and Phe280, were predominant in the recognition of MalE-P11 by mAb164 $(\Delta \Delta G \geq 3.5 \mathrm{kcal} / \mathrm{mol})$. The side chains of Met 282 and Lys 283 were more weakly involved $(1.9<\Delta \Delta G<2.5 \mathrm{kcal} /$ mol). The side chains of His273 and Arg275 were not involved. The three other residues were glycines and had no side chain. These results extend to peptides the observation that the free energy of binding between proteins is generated by a small number of strong interactions, often involving hydrophobic residues, and not by the accumulation 
Table 2: Contributions of Side-Chain Groups to the Energy of Interaction between MalE-P11 and $\mathrm{mAb} 164^{a}$

\begin{tabular}{llrl}
\hline change & \multicolumn{1}{c}{ deleted groups } & \multicolumn{1}{c}{$\begin{array}{c}\Delta \Delta G \pm \mathrm{SE} \\
(\mathrm{kcal} / \mathrm{mol})\end{array}$} & $\begin{array}{c}\langle\Delta \Delta G\rangle \pm \mathrm{SD} \\
(\mathrm{kcal} / \mathrm{mol})\end{array}$ \\
\hline V276A & $\mathrm{C}_{\gamma 1}-\mathrm{H}_{3}, \mathrm{C}_{\gamma_{2}}-\mathrm{H}_{3}$ & $3.8 \pm 0.2$ & $2.5 \pm 0.9$ \\
I278A & $\mathrm{C}_{\gamma 1}-\mathrm{H}_{2}, \mathrm{C}_{\gamma 2}-\mathrm{H}_{3}, \mathrm{C}_{\delta}-\mathrm{H}_{3}$ & $5.1 \pm 0.6$ & $3.8 \pm 0.7$ \\
I278V & $\mathrm{C}_{\delta}-\mathrm{H}_{3}$ & $5.5 \pm 0.1$ & $1.3 \pm 0.4$ \\
V278A & $\mathrm{C}_{\gamma 1}-\mathrm{H}_{3}, \mathrm{C}_{\gamma 2}-\mathrm{H}_{3}$ & $-0.4 \pm 0.6$ & $2.5 \pm 0.9$ \\
Y279A & $\mathrm{C}_{\gamma}$ to $\mathrm{O}_{\eta}-\mathrm{H}$ & $4.6 \pm 0.3$ & $2.7 \pm 2.7$ \\
Y279F & $\mathrm{O}_{\eta}-\mathrm{H}$ & $1.2 \pm 0.6$ & $1.8 \pm 1.2$ \\
F279A & $\mathrm{C}_{\gamma}$ to $\mathrm{C}_{\zeta}-\mathrm{H}$ & $3.4 \pm 0.7$ & $3.8 \pm 0.3$ \\
F279L & $\mathrm{C}_{\epsilon 1}-\mathrm{H}, \mathrm{C}_{\epsilon 2}-\mathrm{H}, \mathrm{C}_{\zeta}-\mathrm{H}$ & $1.2 \pm 0.6$ & na \\
L279A & $\mathrm{C}_{\gamma}-\mathrm{H}, \mathrm{C}_{\delta 1}-\mathrm{H}_{3}, \mathrm{C}_{\delta 2}-\mathrm{H}_{3}$ & $2.2 \pm 0.3$ & $3.5 \pm 1.1$ \\
F280A & $\mathrm{C}_{\gamma}$ to $\mathrm{C}_{\zeta}-\mathrm{H}$ & $1.4 \pm 0.2$ & $3.8 \pm 0.3$ \\
F280G & $\mathrm{C}_{\beta}-\mathrm{H}_{2}$ to $\mathrm{C}_{\xi}-\mathrm{H}$ & $3.5 \pm 0.1$ & $6.3 \pm 0.8$ \\
F280L & $\mathrm{C}_{\epsilon 1}-\mathrm{H}, \mathrm{C}_{\epsilon 2}-\mathrm{H}, \mathrm{C}_{\zeta}-\mathrm{H}$ & $3.6 \pm 0.1$ & na \\
L280A & $\mathrm{C}_{\gamma}-\mathrm{H}, \mathrm{C}_{\delta 1}-\mathrm{H}_{3}, \mathrm{C}_{\delta 2}-\mathrm{H}_{3}$ & $-2.2 \pm 0.1$ & $3.5 \pm 1.1$ \\
A280G & $\mathrm{C}_{\beta}-\mathrm{H}_{3}$ & $2.1 \pm 0.1$ & na \\
M282G & $\mathrm{C}_{\beta}-\mathrm{H}_{2}$ to $\mathrm{C}_{\epsilon}-\mathrm{H}_{3}$ & $2.5 \pm 0.1$ & $4.2 \pm 0.6$ \\
M282A & $\mathrm{C}_{\gamma}-\mathrm{H}_{2}, \mathrm{~S}_{\delta}, \mathrm{C}_{\epsilon}-\mathrm{H}_{3}$ & $1.9 \pm 0.1$ & $3.0 \pm 0.9$ \\
A282G & $\mathrm{C}_{\beta}-\mathrm{H}_{3}$ & $0.6 \pm 0.1$ & na \\
K283M & $\mathrm{N}_{\zeta}-\mathrm{H}_{2}$ & $1.0 \pm 0.2$ & na \\
M283A & $\mathrm{C}_{\gamma}-\mathrm{H}_{2}, \mathrm{~S}_{\delta}, \mathrm{C}_{\epsilon}-\mathrm{H}_{3}$ & $1.2 \pm 0.3$ & $3.0 \pm 0.9$ \\
A283G & $\mathrm{C}_{\beta}-\mathrm{H}_{3}$ & $-0.1 \pm 0.3$ & na \\
\hline
\end{tabular}

${ }^{a}$ F279A, change in the P11 moiety of MalE-P11 replacing a Phe side chain in position 279 by Ala. The value of $\Delta \Delta G$ for $\mathrm{F} 279 \mathrm{~A}$ is given by $\Delta \Delta G(\mathrm{~F} 279 \mathrm{~A})=\Delta G(\mathrm{Y} 279 \mathrm{~F})-\Delta G(\mathrm{Y} 279 \mathrm{~A})$ with the notations of Table 1; its standard error was calculated from the standard errors on $\Delta G(\mathrm{Y} 279 \mathrm{~F})$ and $\Delta G(\mathrm{Y} 279 \mathrm{~A})$ according to the method described in Table 1. $\langle\Delta \Delta G\rangle \pm \mathrm{SD}$, average destabilization of proteins and associated standard deviation for the type of change of column 1 in their hydrophobic core (Shortle et al., 1990; Pace, 1992; Shirley et al., 1992). na, not available.

of numerous weak contacts (Altschuh et al., 1992; Hawkins et al., 1993; Clackson \& Wells, 1995; England et al., 1997).

Mutations of Gly into Ala. Alanine has an additional $\mathrm{C}_{\beta} \mathrm{H}_{3}$ group when compared to glycine, and the $(\phi, \psi)$ torsion angles that an Ala residue can adopt belong to a subset of those that a Gly residue can adopt (Ramachandran \& Sasisekharan, 1968). The destabilizing effects of mutations G274A and G281A on the interaction between MalE-P11 and mAb164 could therefore be due to steric clashes between the mutant side chains and either residues of mAb164 or neighboring residues of P11. They could also be due to a conformational change of P11. The lack of effect of mutation G277A showed that the interaction was compatible with an Ala residue in position 277.

Progressive Deletions of the Side Chains. Several side chains of P11 were progressively deleted to test the contribution of their different groups to the binding of mAb164 (Table 2). The changes of residue Ile278 into Val and Ala showed an important contribution of its $\mathrm{C}_{\delta} \mathrm{H}_{3}$ and no contribution of its $\mathrm{C}_{\gamma 1} \mathrm{H}_{2}$ and $\mathrm{C}_{\gamma 2} \mathrm{H}_{3}$. The changes of Tyr279 into Phe, Leu, and Ala showed an important contribution of the proximal part of its aromatic cycle and slightly less important contributions of the distal part of the cycle and of the hydroxyl group $\mathrm{O}_{\eta} \mathrm{H}$. Mutation F280A affected the binding of mAb164 less strongly than F280L although it deleted the side chain of residue Phe 280 more. This result showed that the side chain of Leu in position 280 created unfavorable interactions that did not exist with the wild type Phe. F280G affected the binding more strongly than F280A (3.5 vs $1.4 \mathrm{kcal} / \mathrm{mol})$, which showed an important contribution of the $\mathrm{C}_{\beta} \mathrm{H}_{2}$ of Phe280. The contributions of various parts of the Met282 and Lys 283 side chains were also analyzed.

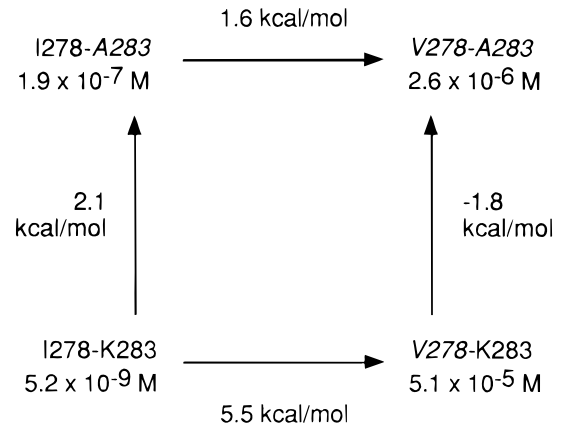

FIGURE 1: Thermodynamic cycle comparing the effects of single and double mutations in MalE-P11 on the energy of interaction with mAb164. The mutant residues are indicated in italic type.

Table 3: Variations in the Energy of Interaction between MalE-P11 and mAb164 for Changes from Ala into Pro ${ }^{a}$

\begin{tabular}{cccc}
\hline change & $\Delta \Delta G \pm \mathrm{SE}(\mathrm{kcal} / \mathrm{mol})$ & change & $\Delta \Delta G \pm \mathrm{SE}(\mathrm{kcal} / \mathrm{mol})$ \\
\hline $\mathrm{A} 276 \mathrm{P}$ & $0.7 \pm 0.3$ & $\mathrm{~A} 280 \mathrm{P}$ & $2.7 \pm 0.2$ \\
$\mathrm{~A} 277 \mathrm{P}$ & 6.4 & $\mathrm{~A} 281 \mathrm{P}$ & $1.6 \pm 0.2$ \\
$\mathrm{~A} 278 \mathrm{P}$ & $-2.0 \pm 0.6$ & $\mathrm{~A} 282 \mathrm{P}$ & $2.2 \pm 0.2$ \\
$\mathrm{~A} 279 \mathrm{P}$ & $1.5 \pm 0.3$ & & \\
\hline
\end{tabular}

Contribution of the Polypeptide Backbone. We changed individually residues $276-282$ into prolines to probe the contribution of the polypeptide backbone of P11 to the recognition by $\mathrm{mAb} 164$. We compared the mutations into Pro with those into Ala to eliminate the effects of the side chains (Table 3). Proline can adopt cis and trans conformations, contrary to the other residues, which adopt only the trans conformation. Proline adopts well-defined $(\phi, \psi)$ dihedral angles and constrains the $(\phi, \psi)$ angles of the residue on its N-terminal side, which adopts an extended conformation in $>90 \%$ of the cases (MacArthur \& Thornton, 1991). In addition, the imide group of a proline is not donor of a hydrogen bond. The values of $\Delta \Delta G$ for mutations $\mathrm{A} 276 \mathrm{P}$ $(0.7 \mathrm{kcal} / \mathrm{mol})$ and A278P $(-2.0 \mathrm{kcal} / \mathrm{mol})$ showed that the recognition between MalE-P11 and mAb164 was compatible with the $(\phi, \psi)$ angles of a trans-Pro at positions 276 and 278 , and therefore suggested with a $>90 \%$ probability that this recognition was compatible with extended conformations of residues Arg275 and Gly277. The solventaccessible surface area and the van der Waals volume of a Pro residue are much smaller than the Tyr, Phe, and Met ones (Miller et al., 1987). As a first approximation, we therefore assume that mutations Y279P, F280P, and M282P did not sterically hinder the interaction between P11 and $\mathrm{mAb} 164$. The $\Delta \Delta G$ values for changes $\mathrm{A} 279 \mathrm{P}, \mathrm{A} 280 \mathrm{P}$, and A282P $(\geq 1.5 \mathrm{kcal} / \mathrm{mol})$ then suggest that the recognition between MalE-P11 and mAb164 was incompatible with the conformational constraints that a Pro residue imposed at positions 279,280 , or 282 or that the peptide $\mathrm{NH}$ group of these residues was engaged in an inter- or intramolecular H-bond (see below).

Tertiary Interactions within P11. To test the existence of long-range tertiary interactions between residues of P11 and the importance of these interactions for the recognition of P11 by mAb164, we constructed double mutations in MalE-P11 (Table 1 and Figure 1). The effects of mutations $\mathrm{I} 278 \mathrm{~V}$ and $\mathrm{K} 283 \mathrm{~A}$ were not additive since the $\Delta \Delta G$ for the double mutation, $3.7 \mathrm{kcal} / \mathrm{mol}$, was less than the sum of the $\Delta \Delta G \mathrm{~s}$ for the two single mutations, $7.6 \mathrm{kcal} / \mathrm{mol}$. The destabilizing effect of $1278 \mathrm{~V}$ was much stronger when the 
side chain in position 283 was Lys than when it was Ala. Mutation K283A was destabilizing when the side chain in position 278 was Ile but stabilizing when it was Val. These results showed that an interaction between the side chains of Ile278 and Lys283 was important for the recognition of MalE-P11 by mAb164 and that mutation K283A partially compensated the effect of $\mathrm{I} 278 \mathrm{~V}$. Thus, P11 was recognized by mAb164 in a loop conformation. In contrast, the effects of mutations V276A and K283A were purely additive.

Energy of the Interaction. The energy of the interaction between MalE-P11 and mAb164, calculated from their dissociation constant, $K_{\mathrm{D}}=5 \mathrm{nM}$, was equal to $11.3 \mathrm{kcal} /$ mol. The sum of the $\Delta \Delta G$ s for the mutations that cut the side chains into Ala was equal to $18.8 \mathrm{kcal} / \mathrm{mol}$. The energy surplus probably came from the fact that $\Delta \Delta G=\Delta G(\mathrm{wt})$ $-\Delta G$ (mut) is generally not equal to the incremental binding energy which is due to the deleted side chain (Fersht, 1988) and from the fact that the mutations had not independent effects (Clackson \& Wells, 1995; Carter et al., 1984; Wells, 1990). This last explanation is exemplified by mutations I278V and K283A.

Comparison of the $\Delta \Delta G$ values obtained in the present work with those obtained in other protein studies enabled us to specify the role of some chemical groups of P11 in the recognition by mAb164 (Table 2 ). The $\Delta \Delta G$ induced by mutation V276A of P11 was close to the maximal destabilization of proteins by a change of Val into Ala in their hydrophobic core. Therefore, most of the side chain of Val276 was involved in the recognition between MalE-P11 and $\mathrm{mAb} 164$, either because it participated in the recognized conformation of P11 or because it directly interacted with mAb164. The $\Delta \Delta G$ induced by $\mathrm{Y} 279 \mathrm{~A}$ was larger than the average destabilization of proteins by this type of change but smaller than their maximal destabilization. Moreover, the $\Delta \Delta G$ s for the changes Y279F, F279A, and L279A were all close to the corresponding average destabilizations of proteins but smaller than them. Thus, Tyr279 brought a large part of the (intra- or intermolecular) interaction energy that the side chain of a tyrosine can bring; this energy was spread over the side chain but it was not optimal. Only part of the interaction energy that the side chains of Phe280, Met282, and Lys 283 could bring were used in the recognition between P11 and mAb164 (Table 2; Schreiber \& Fersht, 1993). The $\Delta \Delta G$ for $\mathrm{K} 283 \mathrm{M}$ was compatible with the removal of a H-bond between the $\mathrm{N} \xi \mathrm{H}_{2}$ of Lys 283 and an uncharged donor or acceptor group (Fersht et al., 1985).

The $\Delta \Delta G$ s for mutations I278V and K283A were strongly dependent on the context (Figure 1). In the context of the wild-type Lys283, the $\Delta \Delta G$ s for mutations I278V and I278A were identical. They were 4 times higher than the average destabilization of proteins by the change of Ile into Val but only 1.3 times higher than this destabilization by the change of Ile into Ala (Table 2). These comparisons indicated that the $\mathrm{C}_{\gamma 1} \mathrm{H}_{3}$ and $\mathrm{C}_{\gamma 2} \mathrm{H}_{3}$ of the mutant Val278 did not replace the $\mathrm{C}_{\gamma 1} \mathrm{H}_{2}$ and $\mathrm{C}_{\gamma 2} \mathrm{H}_{3}$ of the wild-type Ile278; i.e., that mutation $\mathrm{I} 278 \mathrm{~V}$ induced a conformational change of P11 such that the full energy provided by the side chain of Ile 278 was lost. In the context of the mutant Ala283, the $\Delta \Delta G$ for mutation $\mathrm{I} 278 \mathrm{~V}(1.6 \mathrm{kcal} / \mathrm{mol})$ was close to the average destabilization of proteins by this type of change $(1.3 \mathrm{kcal} /$ mol). Conversely, in the context of the mutant Val278, the $\Delta \Delta G$ for mutation $\mathrm{K} 283 \mathrm{~A}(-1.8 \mathrm{kcal} / \mathrm{mol})$ corresponded to a stabilization of the complex between MalE-P11 and
mAb164. The energy restored by K283A, $1.8 \mathrm{kcal} / \mathrm{mol}$, was close to the average destabilization of proteins by a change of Val into Ala $(2.5 \mathrm{kcal} / \mathrm{mol})$. Thus, the side chain of Lys283 appeared to prevent the side chain of Val278 from providing its energy to the interaction with mAb164, and the deletion $\mathrm{K} 283 \mathrm{~A}$ appeared to free this energy.

The $\Delta \Delta G$ s of mutations A279P, A281P, and A282P were compatible with the involvement of the peptide $\mathrm{NH}$ of residues Tyr279, Gly281, and Met282 in a H-bond and its disruption by the change into Pro (Table 3; Fersht et al., $1985)$. In contrast, the $\Delta \Delta G$ s of $\mathrm{A} 277 \mathrm{P}$ and $\mathrm{A} 280 \mathrm{P}$ were larger than the energy of a $\mathrm{H}$-bond and suggested that the effects of these mutations were not solely due to the blocking of the peptide NH group of residues Gly277 and Phe280.

Compatibility with the Structure of Tryptophan Synthase. mAb164 is directed against the free form of protein $\operatorname{TrpB} B_{2}$. A priori, the conformation of $\mathrm{P} 11$ that is recognized by mAb164 could differ from the structure that residues 273283 of $\operatorname{TrpB}$ adopt in the crystals of $\operatorname{Trp} \mathrm{A}_{2} \operatorname{TrpB} B_{2}$, for the three following reasons. P11 is an isolated peptide, out of its normal protein context. $\operatorname{TrpB}_{2}$ undergoes a conformational change when it associates with TrpA (Miles, 1991). Several residues of the segment His273-Lys283 belong to the interface between TrpA and TrpB. Nevertheless, we found that our results on the interaction between MalEP11 and mAb164 were generally compatible with the predictions that can be made from the structure of $\operatorname{Trp} \mathrm{A}_{2}$ $\operatorname{TrpB}_{2}$.

Residues Val276-Lys283 adopt a hairpin conformation in the crystal structure of $\operatorname{Trp}_{2} \operatorname{TrpB}_{2}$ (Figure 2). This structure is compatible with the existence of two $\mathrm{H}$-bonds, between the peptide $\mathrm{N}-\mathrm{H}$ of Tyr279 and $\mathrm{C}-\mathrm{O}$ of Met282 $(\mathrm{N} \cdots \mathrm{O}=2.95 \AA)$ and between the $\mathrm{C}-\mathrm{O}$ of Tyr279 and the $\mathrm{N}-\mathrm{H}$ of Met282 (3.12 $\AA$ ). The distances between the $\mathrm{C}_{\alpha} \mathrm{S}$ of Ile278 and Gly281 (6.91 $\AA$ ) and the $\mathrm{C}_{\alpha} \mathrm{s}$ of Tyr279 and Met282 (5.62 $\AA$ ) are shorter than $7 \AA$. Therefore, residues Ile278-Met282 form a double $\beta$-turn in a 2:2 hairpin. The $\phi$ and $\psi$ angles of residues Tyr279-Phe280-Gly281 show that this double $\beta$-turn is composed of a disordered type II $\beta$-turn followed by a type $\mathrm{I}^{\prime} \beta$-turn. Therefore, it is a type $\beta_{\mathrm{E} \gamma \gamma}$ turn (Wilmot \& Thornton, 1990).

Residue Gly281 of TrpB occupies the fourth position of a $\beta_{\mathrm{E} \gamma \gamma}$ turn in the structure of $\operatorname{Trp} \mathrm{A}_{2} \operatorname{TrpB} \mathrm{B}_{2}$ and there is a strong preference for a Gly residue at this position of a $\beta_{\mathrm{E} \gamma \gamma}$ turn (Wilmot \& Thornton, 1990). Compatibly, mutation G281A had a strong destabilizing effect on the interaction between MalE-P11 and mAb164. The structure of $\operatorname{TrpA}_{2}$ $\mathrm{TrpB}_{2}$ shows contacts between the side chains of Lys 283 and those of Val276 and Ile278 (at $<3.8 \AA$ ). These contacts could stabilize the hairpin conformation of residues 276283 through van der Waals or hydrophobic interactions. Compatibly, the effects of mutations I278V and K283A on the recognition between MalE-P11 and mAb164 showed that an interaction between the side chains of Ile278 and Lys283 was important and that P11 was recognized by $\mathrm{mAb} 164$ in a loop conformation.

As discussed above, proline introduces constrains on the conformation of the polypeptide backbone. We determined the $(\phi, \psi)$ angles of the residues of $\operatorname{TrpB}$ in the structure of $\operatorname{Trp} \mathrm{A}_{2} \operatorname{TrpB} \mathrm{B}_{2}$ and then measured the distance between each of these residues and the closest typical trans-Pro in a Ramachandran plot (Ramachandran \& Sasisekharan, 1968). We found a correlation between these distances and the 

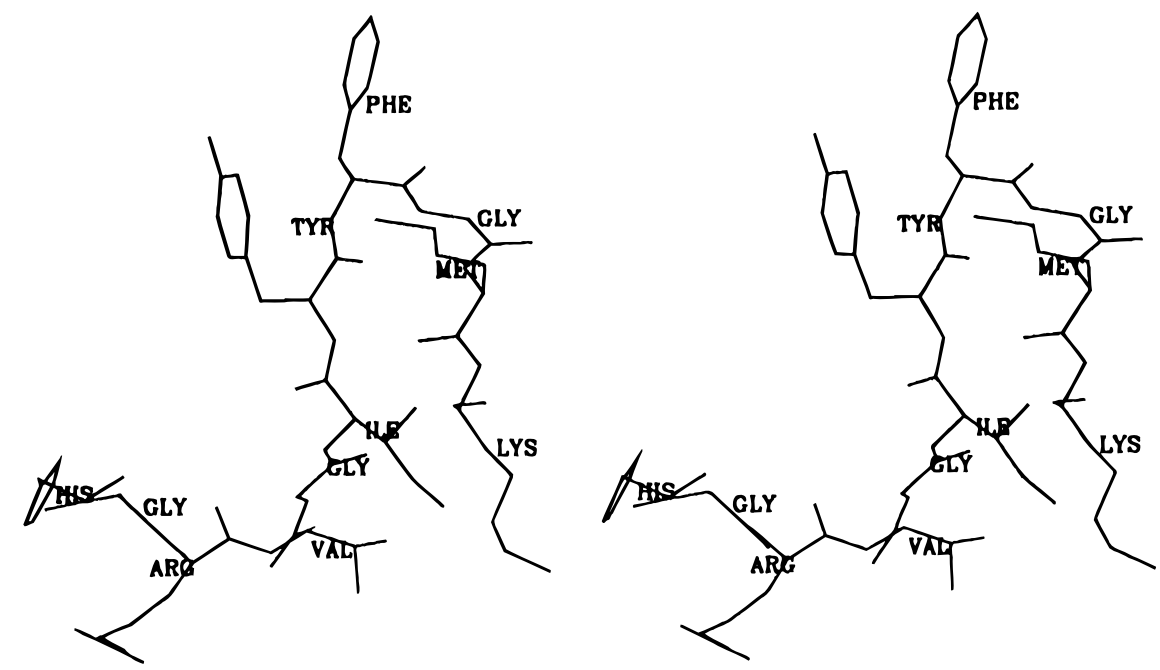

FIGURE 2: Structure of residues $273-283$ of $\operatorname{TrpB}$ in the crystal structure of $\operatorname{Trp}_{2} \mathrm{TrpB}_{2}$ (stereo view).

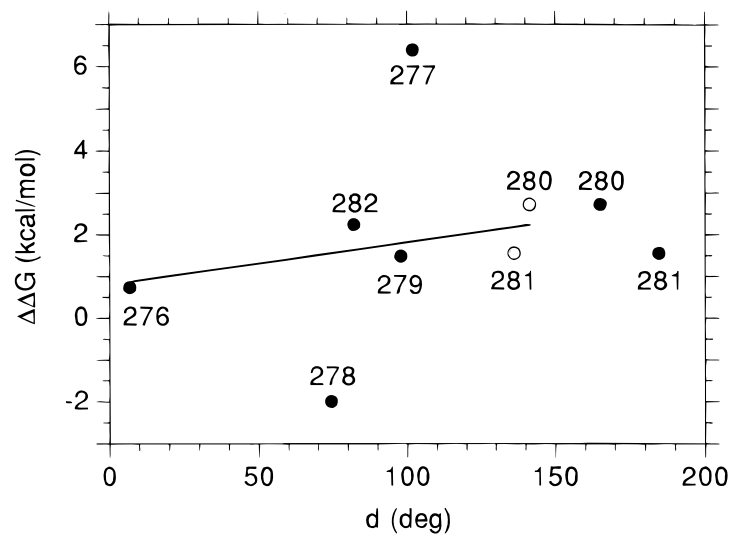

FIGURE 3: Correlation between the $\Delta \Delta G$ s for the changes from Ala into Pro and the predicted variations in the $(\phi, \psi)$ angles. The $\Delta \Delta G$ s for the interaction between MalE-P11 and mAb164 are from Table 3. For each of residues $276-282$ of $\operatorname{TrpB}$, we used the structure of $\operatorname{Trp}_{2} \operatorname{TrpB}_{2}$ to calculate the distance $d$ between its $(\phi$, $\psi$ ) angles and those of a trans-Pro in $(\bullet)$ the $\beta$ region $\left(-65^{\circ}, 150^{\circ}\right)$ or $(O)$ the $\alpha_{\mathrm{R}}$ region $\left(-61^{\circ},-35^{\circ}\right)$, using the relation $d[(\phi, \psi)$, $\left.\left(\phi^{\prime}, \psi^{\prime}\right)\right]=\left\{\left[\left(\phi^{\prime}-\phi\right) \text { modulo } 360^{\circ}\right]^{2}+\left[\left(\psi^{\prime}-\psi\right) \text { modulo } 360^{\circ}\right]^{2}\right\}^{1 / 2}$.

effects of the changes from Ala into Pro on the interaction between MalE-P11 and mAb164, measured as $\Delta \Delta G$ (Figure 3). The coefficient of correlation improved from 0.34 to 0.72 when residues Gly277 and Ile278 were not taken into account. This correlation showed that the effects of the changes from Ala into Pro could be predicted from the structure of $\operatorname{TrpA}_{2} \operatorname{TrpB}_{2}$, except for changes A277P and A278P. The strongly destabilizing effect of A277P suggests that the mutant Pro277 introduced steric clashes with mAb164 rather than conformational constraints on P11. The stabilizing effect of mutation A278P could come from a partial replacement of the Ile side chain, present in the wildtype P11, by the Pro residue. Residues Arg275 and Gly277 have extended conformations in the structure of $\operatorname{Trp}_{2} \mathrm{TrpB}_{2}$, with $(\phi, \psi)$ angles respectively equal to $\left(-114^{\circ}, 138^{\circ}\right)$ and $\left(-158^{\circ},-168^{\circ}\right)$. Compatibly, we found that the changes of A276P and A278P, which introduced a proline residue in position 276 or 278 and thus constrained residue 275 or 277 to adopt an extended conformation, did not prevent the recognition between MalE-P11 and mAb164.

The side chains of Tyr279 and Phe280 have very different conformations in the structure of $\operatorname{Trp}_{2} \operatorname{TrpB}_{2}$ with $\mathrm{Na}^{+}$ bound (Figure 2) and in those with $\mathrm{K}^{+}$or $\mathrm{Cs}^{+}$bound (Rhee et al., 1996). In the structure with $\mathrm{Na}^{+}$bound, the $\mathrm{C}_{\beta} \mathrm{H}_{2}$ group of Phe280 makes Van der Waals contacts with the aromatic cycle of Tyr279 (at $\leq 3.3 \AA$ ), whereas its own aromatic cycle is far from the other side chains of the segment 273-283 of TrpB. In contrast, in the structures with $\mathrm{K}^{+}$or $\mathrm{Cs}^{+}$bound, the aromatic cycles of Tyr279 and Phe280 make several contacts (at $<3.8 \AA$ ) and the $\mathrm{C}_{\beta} \mathrm{H}_{2}$ group of Phe280 is far from the other side chains. We found that the change A280G destabilized the interaction between MalE-P11 and mAb164 by $2.1 \mathrm{kcal} / \mathrm{mol}$ and that mutation F280A affected it less strongly $(1.4 \mathrm{kcal} / \mathrm{mol})$. This comparison between the structure data and our mutagenesis results therefore suggests that the structure of $\operatorname{TrA}_{2} \operatorname{TrpB} B_{2}$ with $\mathrm{Na}^{+}$bound is a better model of the recognized conformation of P11 than those with $\mathrm{K}^{+}$or $\mathrm{Cs}^{+}$bound. It also suggests that the $\mathrm{C}_{\beta} \mathrm{H}_{2}$ group of Phe 280 could be involved in the interaction between MalE-P11 and mAb164 indirectly, by contributing to position the side chain of Tyr279 in the conformation recognized by mAb164.

$\mathrm{mAb} 164$ is directed against the free form of protein $\operatorname{TrpB}_{2}$. If one assumes that the structure of the $\operatorname{TrpB}_{2}$ subunit in the crystals of $\operatorname{TrpA}_{2} \operatorname{TrpB}_{2}$ constitutes a model of the free $\operatorname{TrpB}{ }_{2}$, one can calculate the exposure of each residue of the free $\operatorname{TrpB}_{2}$ to the solvent and thus identify those residues that are potentially available for an interaction with mAb164. Figure 4 relates the solvent-accessible surface area of the groups located beyond the $\mathrm{C} \beta$ (i.e., $\mathrm{C} \gamma, \mathrm{C} \delta, \mathrm{C} \epsilon, \ldots$ ) with the value of $\Delta \Delta G$ when one deletes these same groups through a mutation into Ala. Among the five hydrophobic residues that belong to the segment $273-283$ of TrpB, three have exposed side chains beyond the $\mathrm{C} \beta$ and they were strongly involved in the interaction between MalE-P11 and mAb164 (Val276, Ile278, Tyr279); two have their side chains buried and they were less involved in the interaction (Phe280 and Met282).

Comparison with NMR Data. As mentioned above, the analysis of the isolated P11 by proton NMR has shown that its molecules, in majority, adopt an extended conformation but that some of them, in minority, are structured in their C-terminal part (Delepierre et al., 1991). The analysis of the chemical shifts as a function of $\mathrm{pH}$ has indicated a contact between the side chains of residues Ile278 and Lys283 of P11. The variations of the chemical shifts as a function of temperature, the variations of the corresponding temperature 


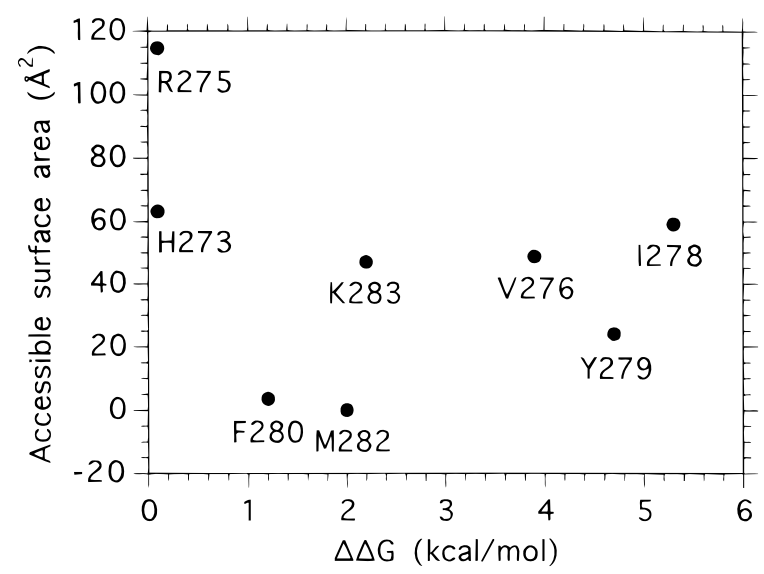

FIGURE 4: Relation between the $\Delta \Delta G$ s for the mutations of the wild-type residues into Ala and the predicted variations in the solvent-accessible surface area of $\operatorname{TrpB}_{2}$. The $\Delta \Delta G$ s for the interaction between MalE-P11 and mAb164 are from Table 1. A structural model of $\mathrm{TrpB}_{2}$ was constructed by removing the atoms of $\operatorname{TrpA}$ in the structure of $\operatorname{Trp}_{2} \operatorname{TrpB}_{2}$. The solvent-accessible surface area for the atoms of a side chain beyond the $\mathrm{C} \beta$ (i.e., $\mathrm{C} \gamma$, $\mathrm{C} \delta, \mathrm{C} \epsilon, \ldots)$ was calculated from this structural model of $\operatorname{TrpB} \mathrm{B}_{2}$ (Materials and Methods).

coefficients as a function of the concentration of urea, and the rates of exchange with the solvent have shown that the NH protons of Tyr279, Met282, and, to a lesser extent, Gly281 are protected from the solvent and that this protection depends on conformation. The authors of the NMR analysis have concluded that molecules of $\mathrm{P} 11$, in small proportion, show some structure around residues Ile278-Lys283, compatible with $\beta$-turns. Compatibly, the double mutation I278V,K283A showed that a contact between Ile278 and Lys283 was important for the recognition between MalE$\mathrm{P} 11$ and mAb164. We also found that the $\Delta \Delta G$ s of mutations A279P, A281P, and A282P were compatible with the involvement of the peptide $\mathrm{NH}$ of residues Tyr279, Gly281, and Met282 in hydrogen bonds.

Implications. The rate constants of dissociation and association between the isolated peptide P11 and mAb164, measured by spectrofluorometry, have a ratio that is equal to the equilibrium dissociation constant between these two molecules, measured by competition ELISA. This equality shows that the binding of P11 to mAb164 does not occur through a mechanism of induced fit (Larvor et al., 1991). Comparison of our mutagenesis results on the recognition between $\mathrm{P} 11$ and mAb164 with the NMR results on the conformation of the free synthetic P11 then suggests that mAb164 selects the molecules of P11, in a small minority, that have the recognized conformation, in a mechanism of "conformational selection" (Leder et al., 1995).

The segment His273-Lys283 of TrpB partially belongs to the interface between $\operatorname{TrpA}$ and $\operatorname{TrpB}_{2}$ since eight of its residues contact $\operatorname{TrpA}$ in the structure of $\operatorname{TrpA} \mathrm{TrpB}_{2}$. Therefore, the dissociation of $\operatorname{TrpA}$ and $\operatorname{TrpB}_{2}$ could result in a conformational change or disorder of this segment. Antibody mAb164 is directed against the free form of $\operatorname{TrpB} B_{2}$. Our results showed that $\mathrm{mAb} 164$ recognized peptide $\mathrm{P} 11$ in a loop conformation that was compatible with that of the segment $273-283$ of $\operatorname{TrpB}$ in the structure of $\operatorname{TrpA} \mathrm{ArpB}_{2}$. Therefore, it is likely that this segment has similar conformations in the free $\operatorname{TrpB} B_{2}$ and in $\operatorname{Trp} \mathrm{A}_{2} \operatorname{TrpB} B_{2}$. To test whether mAb164 recognizes $\mathrm{P} 11$ and $\operatorname{TrpB}_{2}$ through the same mechanism, it will be interesting to compare the effects of the same mutation, introduced into each of the two molecular backgrounds, on this recognition. This comparison could be made with the 30 or so mutations that we have already introduced into MalE-P11.

The properties of the double mutation I278A,K283A of peptide P11 indicated that a short hairpin structure could be stabilized by intramolecular interactions between its side chains. The immunoreactive conformation of P11 could probably be further stabilized and its affinity for antibody mAb164 increased by the introduction of a disulfide bond. This stabilization could help in solving the structure of P11 by NMR spectroscopy. mAb164 and P11 (or hybrid MalEP11) could constitute a valuable experimental system to dissect the parameters on which one can act to stabilize the conformation of a peptide while maintaining or increasing its functional properties.

More generally, the mutational approach that we have developed in this paper to analyze the relationships between the structure of peptide P11, its conformation, and its recognition by mAb164 could be extended to the study of other interactions between peptides and macromolecules.

\section{ACKNOWLEDGMENT}

We thank Dr. Lisa Djavadi-Ohaniance for the gift of antibody mAb164, Dr. Marc Delarue for the calculations of the accessible surface area, and Drs. Sangkee Rhee and David R. Davies for the communication of the refined atomic coordinates of tryptophan synthase with bound $\mathrm{Na}^{+}$, prior to publication.

\section{REFERENCES}

Altschuh, D., Dubs, M.-C., Weiss, E., Zeder-Lutz, G., \& Van Regenmortel, M. H. V. (1992) Biochemistry 31, 6298-6304.

Carter, P. J., Winter, G., Wilkinson, A. J., \& Fersht, A. R. (1984) Cell 38, 835-840.

Clackson, T., \& Wells, J. A. (1994) Trends Biotechnol. 12, 173184.

Clackson, T., \& Wells, J. A. (1995) Science 267, 383-386.

Cortese, R., Monaci, P., Nicosia, A., Luzzago, A., Felici, F., Galfré, G., Pessi, A., Tramontano, A., \& Sollazzo, M. (1995) Curr. Opin. Struct. Biol. 6, 73-80.

Delepierre, M., Larvor, M.-P., Baleux, F., \& Goldberg, M. E. (1991) Eur. J. Biochem. 201, 681-693.

Divita, G., Baillon, J. G., Rittinger, K., Chermann, J. C., \& Goody, R. S. (1995) J. Biol. Chem. 270, 28642-28646.

England, P., Brégégère, F., \& Bedouelle, H. (1997) Biochemistry 36, 164-172.

Fersht, A. R. (1988) Biochemistry 27, 1577-1580.

Fersht, A. R., Shi, J. P., Knill-Jones, J., Lowe, D. M., Wilkinson, A. J., Blow, D. M., Brick, P., Carter, P., Waye, M. M., \& Winter, G. (1985) Nature 314, 235-238.

Friguet, B., Djavadi-Ohaniance, L., \& Goldberg, M. E. (1989a) Res. Immunol. 140, 355-376.

Friguet, B., Djavadi-Ohaniance, L., \& Goldberg, M. E. (1989b) in Protein Structure, A Practical Approach (Creighton, T. E., Ed.) pp 287-310, IRL Press, Oxford, England.

Hawkins, R. E., Russell, S. J., Baier, M., \& Winter, G. (1993) J. Mol. Biol. 234, 958-964.

Houghten, R. A. (1993) Gene 137, 7-11.

Hydes, C. C., Ahmed, S. A., Padlan, E. A., Miles, E. W., \& Davies, D. R. (1988) J. Biol. Chem. 263, 17857-17871.

Koehl, P., \& Delarue, M. (1994) Proteins: Struct., Funct., Genet. 20, 264-278.

Larvor, M.-P., Djavadi-Ohaniance, L., Friguet, B., Baleux, F., \& Goldberg, M. E. (1991) Mol. Immunol. 28, 523-531. 
Leder, L., Berger, C., Bornhauser, S., Wendt, H., Ackermann, F., Jelesarov, I., \& Bosshard, H. R. (1995) Biochemistry 34, 1650916518.

Liuzzi, M., Deziel, R., Moss, N., Beaulieu, P., Bonneau, A. M., Bousquet, C., Chafouleas, J. G., Garneau, M., Jaramillo, J., Krogsrud, R. L., Lagacé, L., McCollum, R. S., Nawoot, S., \& Guindon, Y. (1994) Nature 372, 695-698.

MacArthur, M. W., \& Thornton, J. M. (1991) J. Mol. Biol. 218, 397-412.

Marshall, G. R. (1992) Curr. Opin. Struct. Biol. 2, 904-919.

Miller, S., Janin, J., Lesk, A. M., \& Chothia, C. (1987) J. Mol. Biol. 196, 641-656.

Miles, E. W. (1991) Adv. Enzymol. Relat. Areas Mol. Biol. 64, 93172.

Pace, C. N. (1992) J. Mol. Biol. 226, 29-35.

Ramachandran, G. N., \& Sasisekharan, V. (1968) Adv. Protein Chem. 23, 283-438.

Rhee, S., Parris, K. D., Ahmed, S. A., Miles, E. W., \& Davies, D. R. (1996) Biochemistry 35, 4211-4221.
Rondard, P., Brégégère, F., Lecroisey, A., Delepierre, M., \& Bedouelle, H. (1997) Biochemistry 36, 8954-8961.

Schreiber, G., \& Fersht, A. R. (1993) Biochemistry 32, 5145-5150.

Scott, J. K., \& Smith, G. P. (1990) Science 249, 386-390.

Shirley, B. A., Stanssens, P., Hahn, U., \& Pace, C. N. (1992) Biochemistry 31, 725-732.

Shortle, D., Stites, W. E., \& Meeker, A. K. (1990) Biochemistry 29, 8033-8041.

Shrake, A., \& Ruplay, J. A. (1973) J. Mol. Biol. 79, 351-371.

Stanfield, R. L., \& Wilson, I. A. (1995) Curr. Opin. Struct. Biol. $5,103-113$.

Stevens, F. (1987) Mol. Immunol. 24, 1055-1060.

Wells, J. A. (1990) Biochemistry 29, 8509-8517.

Wilmot, C. M., \& Thornton, J. M. (1990) Protein Eng. 3, 479493.

Zvelebil, M. J. J. M., \& Thornton, J. M. (1993) Q. Rev. Biophys. 26, 333-363.

BI9625096 